\title{
AS REDES SOCIAIS NO PROCESSO DE DIFUSÃO DA INFORMAÇÃO: DO MODELO DE GRANOVETTER AO DE FLACHE-MACY
}

\author{
Luis Soczka
}

Resumo: Em qualquer dos campos da actividade humana coloca-se sempre o problema dos canais de difusão de informação e de adopção de inovações. Ora, a questão da difusão de inovações liga-se de perto ao tema do sistema de ciência, e não só na vertente de difusão de produtos tecnológicos, já que as inovações não têm necessariamente de ser produtos materiais. Podem muito bem ser ideias, conceitos, hipóteses, modelos, teorias. Neste trabalho, o autor aborda o modelo da força das ligações fracas de Granovetter e da difusão em rede social das inovações de Flache-Macy, sustentando que as abordagens mais convincentes das dinâmicas de difusão se situam na encruzilhada da Psicologia Social com a Análise de Redes Sociais, já que tudo indica que a difusão opera através de redes sociais. O problema não está em ver os estudos de influência social como estratégicos para o estudo de redes sociais, mas justamente o contrário: encarar as redes sociais como uma arena estratégica para o estudo dos processos de influência social.

Palavras-chave: redes sociais, processo de difusão da informação, modelo de Granovetter, modelo de Flache-Macy

Social networks in the process of diffusion of information: From Granovetter's model to the model of Flache-Macy (abstract): In any facet of human activity the problem of the channels for innovation diffusion and information transmission must be dealed with. Innovation diffusion is a concept directly linked to the science system and not only to technological or market objects, since innovation objects may also be ideas, new models or theories. In this work the author analyze Granovetter's "strength of weak ties" and Flache-Macy's social network diffusion models, putting forward that diffusion operates through social networks and those must be seen as the strategic arena for the analysis of social influence studies in the science system.

\footnotetext{
${ }^{1}$ Professor Catedrático da Escola Superior de Altos Estudos, ISMT. 
Neste artigo, o autor visa demonstrar a tese central de que o campo de intersecção entre a Psicologia Social e a Análise de Redes Sociais é, por excelência, a arena estratégica para o estudo dos processos de influência social na difusão da informação. Mostra-se a complementaridade do modelo de Granovetter ("a força dos elos fracos") e o de Flache-Macy ("a fraqueza dos elos fortes"), salientando o papel central das redes sociais nos canais de difusão e abordando as estratégias dessa difusão das inovações.

Em qualquer dos campos da actividade humana coloca-se sempre o problema dos canais de difusão de informação e de adopção de inovações. Ora, este tema da difusão de inovações é bem conhecido no domínio económico e, em marketing, apresenta especial e óbvio interesse. A experiência acumulada sugeriu a adopção no domínio do marketing de modelos orientados pela curva de Bass (1969), equacionável por:

$$
\mathrm{N}_{\mathrm{t}}=\mathrm{N}_{\mathrm{t}-1}+\mathrm{p}\left(\mathrm{m}-\mathrm{N}_{\mathrm{t}-1}\right)+\mathrm{q}\left(\mathrm{N}_{\mathrm{t}-1} / \mathrm{m}\right)\left(\mathrm{m}-\mathrm{N}_{\mathrm{t}-1}\right)
$$

Onde $\mathrm{m}=$ potencial de mercado (número total de entidades ou pessoas que poderão usar o produto); $\mathrm{p}=$ coeficiente de influência externa (probabilidade de alguém que não usa ainda o produto vir a usá-lo por efeito da publicidade ou outros factores externos); $q=$ coeficiente de influência interna (probabilidade de alguém que não usa ainda o produto vir a usá-lo por referência directa de alguém que já o usa). Ou seja: uma função sigmóide, correspondendo o eixo das ordenadas a $\mathrm{N}$ (número de adoptantes do produto) e o das abcissas a $\mathrm{T}$ (tempo decorrido desde a introdução do produto; ver Figura 1):

Figura 1: Função sigmóide

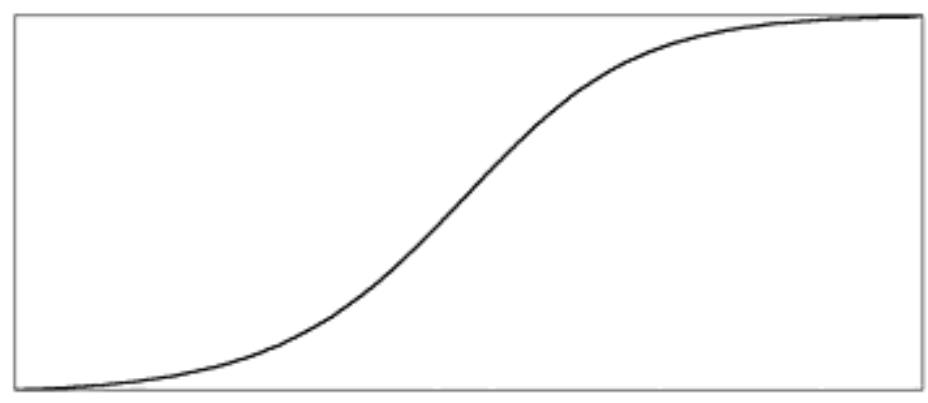


No entanto, múltiplos casos se verificam em que a equação acima não é compatível com a realidade. Por exemplo, o caso da adopção de hipertexto corresponde empiricamente a uma curva descrita por:

$$
\mathrm{N}_{\mathrm{t}}=\mathrm{N}_{\mathrm{t}-1}+\mathrm{p}\left(\mathrm{m}-\mathrm{N}_{\mathrm{t}-1}\right)+\mathrm{q}\left[\left(\mathrm{N}_{\mathrm{t}-1}\right) / \mathrm{m}\right]\left(\mathrm{m}-\mathrm{N}_{\mathrm{t}-1}\right)
$$

conforme o demonstrou Nielsen (1995). Neste caso, o impacte de outrém na adopção do hipertexto não é simplesmente proporcional a $\mathrm{N}$ e indica um crescimento que não é simplesmente linear. Segundo os cálculos de Nielsen, em 1995, 1\% da população do mundo industrializado usaria a Web, mas 5\% seriam utilizadores de hipertexto por outras vias (bases de dados, CD-ROMs, etc.). Acresce que esse parâmetro não é, ainda por cima, uniforme, nem no tempo nem no espaço. Tome-se o caso do boom de utilização de telemóveis em Portugal, por exemplo, que excedeu todas as previsões dos próprios operadores e abriu caminho à rápida implantação de um terceiro operador face à apetência do mercado.

Seja como for, o modelo de Bass pressupõe uma assimptota no tempo que não é compatível com o problema da substituição das ofertas. Não é necessário atingir um ponto de saturação da procura de um produto inovador para que se verifique a sua substituição. Este problema foi bem assinalado por Maier, da Universidade de Mannheim (1995) e um dos casos mais flagrantes é o dos processadores da Intel, que, na sua rapidíssima evolução, desactualizam de um dia para o outro os produtos antes oferecidos como topo de gama pela própria Intel: um Pentium II a $450 \mathrm{MHz}$, topo de gama de 1998, veio destronar os Pentium I de 1996-97 a $200 \mathrm{MHz}$, que, por sua vez, afastaram do mercado os 80486 , hoje desactualizados e desvalorizados ainda no primeiro semestre de 1999 pelos processadores Pentium III, a $600 \mathrm{MHz}$, e os Pentium IV contam actualmente com processadores da ordem de velocidade dos GigaHertz, etc.

A figura seguinte, retirada do citado trabalho de Maier (1995), ilustra bem o caso da substituição de processadores Intel entre 1984 e 1996 (ver Figura 2):

Ora, a questão da difusão de inovações liga-se de perto ao tema do sistema de ciência, e não só na vertente de difusão de produtos tecnológicos, já que as inovações não têm necessariamente de ser produtos materiais. Podem muito bem ser ideias, conceitos, hipóteses, modelos, teorias. E, neste aspecto, é bem conhecido o conservadorismo dos cientistas, muito superior no domínio dos modelos e teorias do que no domínio da adopção de novos produtos tecnológicos. Há verdadeiros dinossauros do pensamento que nem por isso deixam de ser ávidos para experimentar as mais recentes tecnologias. Mas as ideias não são como os processadores da Intel: têm um tempo de vida muitíssimo mais longo, podem ter a idade do discurso de Buda na Montanha, dos Rigvedda, dos diálogos de Platão, da 
Bíblia, da Utopia de Moore. E, mesmo no domínio científico, as leis de Newton ou o Traumdeutung de Freud ainda têm hoje muitíssima mais actualidade e "procura" do que os processadores a $33 \mathrm{Mhz}$. É natural, portanto, esse aspecto de conservadorismo científico e incorpora-se no que Kuhn designou por ciência normal, na sua teoria dos paradigmas da ciência.

Figura 2: Substituição de processadores Intel entre 1984 e 1996

\author{
Inserir figura 2 (fig0210a imagem de \\ mapa de bits) mais ou menos aqui
}

Mas, no entanto, ela mexe-se. Em domínios como o das ciências biomédicas, mexe-se mesmo a uma velocidade vertiginosa. O problema que se põe é, pois, não o da mudança ou conservação em si mesmas consideradas, mas o dos canais e forças que operam num ou noutro sentido, na comunidade científica. As abordagens mais convincentes das dinâmicas de difusão situam-se na encruzilhada da psicologia social com a $\mathrm{ARS}^{2}$, já que tudo indica que a difusão opera através de redes sociais (Coleman, Katz e Menzel, 1966; Roger e Kincaid, 1981; Granovetter, 1982; Burt, 1987; Marsden e Friedkin, 1994; Valente, 1995). No terreno da Psicologia Social, a difusão de inovações liga-se estreitamente ao problema dos processos de

2 ARS - Análise de Redes Sociais, família de modelos matemáticos para o estudo da estrutura e configuração das redes sociais (cf., Soczka, 2001). 
influência social, o que não significa que a Psicologia Social contemporânea tenha grandemente contribuído para compreender os processos de difusão, dada a invalidade ecológica de muitos dos mais afamados estudos sobre a influência social. Os estudos experimentais realizados em laboratório estão à partida viciados por invalidade ecológica (Soczka, 1989, 2004) e não têm em linha de conta os contextos de rede social dos cenários ambientais reais. E, no entanto, continuam a ser os estafados estudos experimentais de Sheriff, Ash e outros similares os que são apresentados como modelos em handbooks de Psicologia Social. Portanto, a meu ver, o problema não está em ver os estudos de influência social como estratégicos para o estudo de redes sociais, como o pretendem Marsden e Friedkin (1994), mas justamente o contrário: encarar as redes sociais como uma arena estratégica para o estudo dos processos de influência social. Em primeiro lugar, a maioria dos estudos sobre os processos de influência social assentam em modelos de influência interpessoal ou intergrupal que recorrem a teorias de comparação e categorização social, tal como, já há mais de trinta anos, Cartwight (1965) tinha chamado a atenção para aquilo que designou por influência ecológica: a transformação de atitudes, representações ou acções de um indivíduo ou grupo de indivíduos, devido não a relações interpessoais ou intergrupais directas, mas simplesmente a mudanças das condições ambientais. Considere-se, por exemplo, um caso de influência ecológica como o da transformação de atitudes e até de representações identitárias na Musgueira Norte, em função do realojamento de barracas para casas de habitação social (Soczka et al., 1986, 1989). O mesmo acontece no caso do efeito de propinquidade de Festinger, estudado por Soczka e colaboradores (1985) e por Borges (1993) em bairros degradados da cidade de Lisboa.

Grosso modo, os modelos de influência podem dividir-se (Valente, 1995) em duas grandes famílias: 1) os modelos relacionais, onde a influência é exercida através de ligações directas numa rede; 2) e os modelos estruturais, em que as influências são veiculadas através das posições dos elementos no sistema social. Neste último caso, incluem-se as influências por equivalência estrutural (Burt, 1987; Lorrain e White, 1971). Um exemplo de movimento coordenado por uma similaridade de posições em equivalência estrutural seria a acção conjunta dos "capitães" no desencadear do 25 de Abril. Um modelo simples e linear de influência em rede poderia ser representado por

$$
\mathrm{y}_{\mathrm{t}+1}=\mathbf{W}_{y_{\mathrm{t}}}
$$

isto é: sendo $\mathbf{y}$ um vector contendo as atitudes das unidades da rede, os seus valores no tempo $t+1$ são função do produto de $\mathbf{W}$ (a matriz dos coeficientes de influência) pelo vector das atitudes $\mathbf{y}$ no tempo t. Como o no- 
tam Marsden e Friedkin (1994), se W contiver apenas valores positivos e normalizados de forma a que

$$
\sum_{j=1}^{n} w_{i j}=1
$$

para o elemento $i$, e para $i \neq j$, então

$$
y_{i, t+1}=y_{i t}+\sum_{j=1}^{n} w_{i j}\left(y_{j t}-y_{i t}\right)
$$

as mudanças de atitudes sendo assim função da interação entre as relações de influência e as discrepâncias de atitudes.

Friedkin e Johnsen (1990, citados por Marsden \& Friedkin, 1994) generalizaram este modelo linear introduzindo covariantes tais que as atitudes iniciais $\mathbf{y}_{0}$ têm um efeito persistente em $\mathbf{y}_{t}$,

$$
\begin{aligned}
& \mathbf{y}_{0}=\mathbf{X} \boldsymbol{b} \\
& \mathbf{y}_{\mathrm{t}+1}=\alpha \mathbf{W} \mathbf{y}_{\mathrm{t}}+\beta \mathbf{X} \boldsymbol{b}
\end{aligned}
$$

em que $\mathbf{W}$ é uma matriz de covariação a nxp elementos e $\boldsymbol{b}$ é um vector de coeficientes de regressão das covariâncias, sendo os parâmetros $\alpha$ e $\beta$ pesos para as componentes endógenas e exógenas das atitudes. No mesmo trabalho, é criado um modelo de equilibrio das atitudes segundo o modelo acima, tal que:

$$
\mathbf{y}_{\mathrm{e}}=(\boldsymbol{I}-\alpha \mathbf{W})^{-1} \beta \mathbf{X} b=\alpha \mathbf{W} \mathrm{y}_{\mathrm{e}}+\beta \mathbf{X} b .
$$

Para escapar ao carácter determinístico do modelo, Marsden e Friedkin (1994) introduziram um parâmetro de erros estocásticos $\varepsilon$, de média 0 e matriz de covariância $\alpha_{\varepsilon}^{2} \boldsymbol{I}$, o que nos conduz a:

$$
\mathbf{Y}=\alpha \mathbf{W y}+\mathbf{X} \beta+\varepsilon,
$$

designando este modelo por "modelo de efeitos de rede".

Dada a ausência de qualquer estudo sistemático sobre os canais de influência e difusão na rede científica nacional, a questão que se põe é: processar-se-ão elas mais por via de conexões directas ou por via de homofilias e equivalências estruturais? A hipótese mais plausível é a de que não 
será possível encontrar um padrão homogéneo, que dentro do mesmo subsistema encontraremos grandes oscilações entre um e outro modelo e que isso encontrará grande variação, de acordo com o subsistema específico em análise.

Uma outra questão relacionada com a anterior prende-se com a verificação da hipótese da força dos elos fracos de Granovetter. Em trabalhos hoje célebres, Granovetter $(1973,1982)$ chamou a atenção para o papel das influências em grupos de fraca densidade $\Delta$, onde os actores sociais estão nodalmente distantes e as redes têm uma coesão débil. Esses grupos instrumentais apresentam uma interligação afectiva muito menor do que outros, tais como as famílias, os grupos militares ou religiosos. E, no entanto, proporcionam um melhor fluxo de trocas e uma mais eficiente e rápida difusão de inovações. Os estudos clássicos de dinâmica de grupos apontam para uma conclusão comum: quanto maior for a coesão verificada, maior a adesão dos membros do grupo às normas, ou seja, a conformidade dos membros do grupo é maior (modelo de coesão-conformidade de Homans, 1951). A hipótese demonstrada por Granovetter incide justamente sobre grupos de fraca coesão, em que se verificam laços fracos que são, por isso mesmo, veículos de difusão de inovação. Dado que o efeito de conformidade facilita uma homogeneidade da informação, estes grupos reflectem, portanto, não só uma ausência de novidade como também uma resistência a novas informações e a mudanças. Logo, menos permeabilidade a inovações, a bem do equilíbrio estrutural da rede e do evitamento de dissonâncias cognitivas.

Flache e Macy (1997) produziram um interessante trabalho que visa a parte mais esquecida da hipótese de Granovetter e que dela decorre: se é verificável e plausível a "força dos elos fracos", então seria também verdade o seu complemento, a fraqueza dos elos fortes. Procederam então à construção de um modelo matemático que simularam computacionalmente, e que será seguidamente exposto, seguindo tão de perto quanto possível os passos do modelo de Flache-Macy.

O modelo de trocas sociais de Homans, em que a coesão é medida pelo grau de atracções interpessoais e os membros do grupo se gratificam reciprocamente trocando conformidade às normas por aprovação $(\mathrm{C}-\mathrm{A})$, influenciou muita da investigação sobre o controlo social. Flache e Macy sublinham alguns pontos mais frágeis deste modelo de controlo social, dado que se é plausível que a aprovação pelos membros do grupo seja a moeda de troca da conformação às normas do grupo, já não é tão óbvio que a aprovação seja por sua vez trocada pela conformação. A aprovação pode ser trocada apenas por aprovação e as trocas não têm necessariamente de ser equitativas. Dois vectores podem, portanto, ser diferenciados: a) numa rede social, os actores podem estabelecer laços para fortalecer as confor- 
midades às normas do grupo (controlo de conformidade); b) ou para fortalecer relações interpessoais satisfatórias (controlo relacional). A esta última situação chamaram Flache e Macy "cohesion-resistance hypothesis". A troca recíproca de aprovações (A-A) pode, então, comprometer a eficácia do controlo social informal: "Instead of giving Ego leverage over Alter's compliance, a mutually beneficial exchange may instead give Alter leverage over Ego 's willingness to enforce conformity with group obligations" (Flache e Macy, 1997, p. 21).

O modelo de Flache-Macy coloca, assim, um problema de vantagens oportunistas de segunda ordem (second-order free-rider problem; Oliver, 1980). A vantagem oportunista de primeira ordem consiste em beneficiar de bens comuns cujo custo foi pago por outros. Flache e Macy notam que as próprias sanções para evitar esta vantagem oportunista também implicam custos, pelo que uma vantagem oportunista de segunda ordem consiste em tirar partido das próprias acções destinadas a sustentar o controlo social, deixando que outros suportem os custos dessa pressão. A introdução da troca da aprovação por aprovação (A-A) coloca-nos perante um paradoxo: por um lado, aumenta a indução da conformidade grupal, mas, por outro, faz diminuir a pressão sobre os oportunistas que satisfaçam a condição de troca A-A mas não invistam na troca de conformidade por aprovação (A-C, modelo de Homans). E é aqui que situa em pleno a hipótese central do modelo de Flache-Macy: se o que prevalecer for a necessidade de trocar aprovações por aprovações, estamos perante uma situação grupal de laços interpessoais fortes, que desencoraja a utilização dos laços afectivos como veículo de mera pressão normativa. Ou seja, a "fraqueza" dos laços fortes. Os próprios autores fazem notar que no seu modelo afastaram o que chamam a "hipótese do narcisismo". Isto é, para um dado momento $t$ existir a relação transitiva $\mathrm{a}_{\mathrm{iit}}=1$. Foi, portanto, mantida nula e afastada a hipótese de o sujeito $i$ ser a sua própria fonte de aprovação social ${ }^{3}$.

Designem-se, então, dois vectores distintos, $\boldsymbol{w}$ e $\boldsymbol{a}$ (correspondentes a trabalho $\mathrm{e}^{\mathrm{e}}$ aprovação, respectivamente) e $\mathrm{p}_{\mathrm{it}}$ a probabilidade de $i$ trabalhar no tempo $\mathrm{t}\left(\mathrm{w}_{\mathrm{it}}=1\right)$, com a probabilidade $1-\mathrm{p}_{\text {it }}$ de se furtar ao trabalho. $\mathrm{E}$ $\mathrm{p}_{\text {ijt }}$ a probabilidade de $i$ aprovar $j$ no tempo $\mathrm{t}\left(\mathrm{a}_{\mathrm{ijt}}=1\right)$, e $1-\mathrm{p}_{\mathrm{ijt}}$ a probalidade de não aprovar.

\footnotetext{
${ }^{3}$ De um ponto de vista totalmente distinto do da ARS, a hipótese faz sentido, porque mesmo numa patologia narcísica, Narciso não é ninguém sem um espelho que o reflicta. No olhar de Alter, Narciso vê-se a si mesmo ao espelho. Mas esse olhar é-lhe necessário. Porque, ao mesmo tempo que nega o outro como outro, outrem é-lhe indispensável para o culto de si mesmo. Aceite-se, portanto, de boa vontade $\mathrm{a}_{\mathrm{iit}}=0$.

4 Entenda-se aqui por "trabalho" qualquer esforço orientado para a produção de um bem comum.
} 
Agregando para o grupo e assumindo equidistribuição de benefícios em que cada actor recebe $1 / \mathrm{N}$ avos dos benefícios do grupo,

$$
W_{t}=\frac{1}{N} \sum_{i=1}^{N} w_{i t}
$$

onde $\mathrm{w}_{\mathrm{it}}=1$ para os contribuintes e $\mathrm{w}_{\mathrm{it}}=0$ para os "baldas".

Similarmente, para a aprovação social,

$$
A_{i t}=\beta \sum_{i=1}^{N} a_{i j t}
$$

onde $\beta$. representa um parâmetro arbitrário do valor de uma unidade de $\boldsymbol{a}$ em relação a uma unidade de $\boldsymbol{w}$.

Flache e Macy estabelecem então modelos para outras duas funções: a função de recompensa e a função de aprendizagem. Aplicando um modelo de racionalidade de decisões, os autores vão pressupor que a satisfação com uma decisão tomada aumenta com a recompensa recebida, avaliada proporcionalmente às recompensas globais no grupo, e diminui em função dos custos envolvidos. E que os actores tomam em atenção o efeito dos seus esforços nas relações com os restantes actores. Altos níveis de aprovação podem conduzir a um mínimo de esforço, dado poderem ser considerados já satisfatórios. $E$ formalizam a satisfação $S_{i t}$ :

$$
\mathrm{S}_{\mathrm{it}}=\mathrm{W}_{\mathrm{t}}+\mathrm{A}_{\mathrm{it}}-\mathrm{cW}_{\mathrm{it}}-\mathrm{S}_{\mathrm{e}}
$$

sendo $\mathrm{S}_{\mathrm{e}}$ uma variável de cálculo de risco, postulando $\mathrm{S}_{\mathrm{e}}=0.5(1+\beta(\mathrm{N}-1)-\mathrm{c})$.

No que respeita à aprovação, o processo é similar, com a diferença de desagregarem a acção colectiva numa matriz de dilemas do prisioneiro, um para cada possível relação diádica. Neste modelo, cada actor calcula o benefício recebido do trabalho de outrem e o benefício recebido da aprovação por outrem, pesa-os contra os custos c' da aprovação e avalia-os contra as expectativas $\mathrm{S}_{\mathrm{e}}$ :

$$
\mathrm{S}^{\prime}{ }_{\mathrm{ijt}}=(1 / \mathrm{N}) \mathrm{w}_{\mathrm{jt}}+\beta^{\prime} \mathrm{a}_{\mathrm{ijt}}-\mathrm{c}^{\prime} \mathrm{a}_{\mathrm{ijt}}-\mathrm{S}_{\mathrm{e}}
$$

onde $\beta$ ' representa o peso dado por $i$ à aprovação de $j$, postulando $\mathrm{S}^{\prime}{ }_{\mathrm{e}}=0.5\left((1 / \mathrm{N})+\beta^{\prime}-\mathrm{c}^{\prime}\right)$. 
Cada decisão é avaliada e transformada num reforço positivo ou negativo no intervalo $[-1,+1], \mathrm{R}_{\mathrm{it}}=\boldsymbol{l} \mathrm{S}_{\mathrm{it}} / \mathrm{S}_{\max }$, sendo $\boldsymbol{l}$ um parâmetro de aprendizagem que pondera a magnitude do reforço e $S_{\max }$ o maior valor possível da avaliação de $\boldsymbol{w}$. Como sublinham os autores do modelo, $\mathrm{S}_{\max }$ requer que todos o actores trabalhem a aprovem $i$, enquanto $i$ assume a estratégia oportunista de não desenvolver trabalho.

$$
\mathrm{S}_{\max }=0.5[(\mathrm{~N}-2 / \mathrm{N})-\beta(\mathrm{N}-1)+\mathrm{c}] .
$$

Analogamente,

$\mathrm{R}_{\mathrm{ijt}}^{\prime}=\left\{2 \boldsymbol{l} \mathrm{S}_{\mathrm{ijt}}^{\prime} /\left[(1 / \mathrm{N})+\beta^{\prime}+\mathrm{c}^{\prime}\right]\right\}$.

A função de aprendizagem adoptada por Flache e Macy recorre a modelos clássicos de aprendizagem estocástica, em que $p_{i t}$ é reforçada quando $\mathrm{w}_{\mathrm{it}}=1$ e $\mathrm{S}_{\mathrm{it}}>0$ (o esforço é recompensado) ou quando $\mathrm{w}_{\mathrm{it}}=0$ mas $\mathrm{S}_{\mathrm{it}}<0$ (o preço da "balda" é muito elevado):

$$
\mathrm{Pi,t}+1=\mathrm{p}_{\mathrm{it}}+\mathrm{R}_{\mathrm{it}}\left(1-\mathrm{p}_{\mathrm{it}}\right) \mathrm{w}_{\mathrm{it}}-\mathrm{R}_{\mathrm{it}}\left(1-\mathrm{p}_{\mathrm{it}}\right)\left(1-\mathrm{w}_{\mathrm{it}}\right)
$$

Sendo $\mathrm{P}^{\prime}{ }_{\mathrm{ijt}}$, referente à aprovação de $i$ por $j$ no tempo t, segue-se o mesmo processo. Se $\mathrm{a}_{\mathrm{ijt}}=1$ e $\mathrm{S}{ }_{\mathrm{ijt}}>0$ ou $\mathrm{a}_{\mathrm{ijt}}=0$ e $\mathrm{S}{ }_{\mathrm{ijt}}<0$, então:

$$
p^{\prime}{ }_{i j t+1}=p^{\prime}{ }_{i j t}+R^{\prime}{ }_{i j t}\left(1-p^{\prime}{ }_{i j t}\right) a_{i j t}-R^{\prime}{ }_{i j t}\left(1-p^{\prime}{ }_{i j t}\right)\left(1-a_{i j t}\right) \text {. }
$$

Finalmente, são calculadas medidas agregadas (relativas à rede social no seu todo) de coesão e conformidade de grupo: $r_{c a, t}=\rho\left(\mathrm{p}_{+\mathrm{i}, \mathrm{t}} \mathrm{p}_{\mathrm{it}}\right)$, controlo de conformidade, "refere-se à tendência para aprovar apenas os actores que trabalham" e é medida pela correlação entre a propensão de $i$ para trabalhar e o somatório das aprovações recebidas. Paralelamente, $r_{a a, t}$ $=\rho\left(\mathrm{p}^{\prime}{ }_{\mathrm{ijt}} \cdot \mathrm{p}\right.$ ' $\left.\mathrm{ijt}\right)$, controlo relacional, refere-se à "tendência para aprovar apenas os actores que retribuem a aprovação", é medido pela correlação entre p' ${ }_{\mathrm{ijt}}$ e p' ${ }_{\mathrm{jit}}$, para todas as díades da rede (Flache e Macy, 1997).

Os resultados obtidos apontam para a fraqueza dos elos fortes: pressão para a conformidade e investimento relacional variam no sentido inverso, e a pressão para a conformidade cede o passo perante os graus elevados de satisfação nas relações interpessoais e coesão entre os membros da rede. Ou seja, a coesão é contrária ao conformismo nas situações em que as aprovações são trocadas por aprovações e não por comportamentos de conformismo às normas de grupo. 
Mais uma vez estamos talvez confrontados com o eterno problema da validade ecológica dos modelos de laboratório que serviram de base às teorias da influência e da conformidade de grupo em Psicologia Social. Pela simples razão de que, nesses casos, os grupos eram constituídos por estranhos entre si, sem laços afectivos recíprocos e, portanto, sem coesão. Daí a facilidade de se chegar a resultados positivos de influência e de conformismo. Parafraseando o célebre dito de Brofenbrenner em relação à Psicologia do Desenvolvimento, a Psicologia Social experimental é o estranho estudo de adultos estranhos entre si a realizarem tarefas que lhes são estranhas, num ambiente que lhes é estranho, impostas em nome de uma estranha ciência por um outro estranho adulto com o qual nada têm a ver.

O modelo de Flache e Macy não contempla explicitamente a existência de metanormas impeditivas de processos oportunistas de segunda ordem, ou seja, situações em que a aprovação de $j$ por $i$ depende de um terceiro actor $k$ externo à rede, ou de uma rede $\mathbf{G}_{2}$ externa à rede em análise $\mathbf{G}_{1}$.

Para o caso que nos interessa aqui, é óbvio que existem metanormas para qualquer subsistema da comunidade científica, se bem que não seja evidente que essas metanormas sejam unívocas, existindo por hipótese maior variabilidade de critérios acerca das metanormas à medida que os paradigmas epistemológicos se afastam dos campos nomotéticos para os campos ideográficos. A pressão para a conformidade pode ser máxima em corpos paradigmáticos com critérios fechados acerca do que é "científicamente correcto". Nesses casos, a cumplicidade na adesão a metanormas comuns pode ser compatível com o que chamaria "pseudo-coesão oportunista", dado que não estabelecida em termos de relações interpessoais, mas em termos de vantagens para $i$ de obter aprovações de $k$ veiculadas através de $j$, seja qual for $j$. Porque $j$ não existe por si próprio, é apenas um veículo de $k$, que não faz parte directamente da rede. Neste caso, $j$ é perfeitamente substituível por qualquer outro que também veicule $k$. Nesses casos, estamos perante sistemas ortodoxos regulados por doxemas (Déconchy) de alto nível, rígidos e apresentando um elevado grau de impermeabilidade grupal, verdadeiramente congruentes com o modelo de Homans C-A, com uma máxima pressão para a conformidade com a metanorma, que se sobrepõe às ligações interpessoais.

\section{Referências}

Bass, F. M. (1969). A new product growth model for consumer durables. Management Science, 15, 215-227. 
Borges, M. J. (1994). Mudança em perspectiva: Redes psico-sociológicas e efeito de propinquidade no Bairro da Liberdade. Monografia de licenciatura em Psicologia Social não publicada. Lisboa: ISPA

Burt, R. S. (1987). Social contagion and innovation: Cohesion versus structural equivalence. American Journal of Sociology, 92, 1287-1335.

Cartwrigth, D. (1965). Influence, leadership, control. In G. March (Ed.), Handbook of organizations (pp. 1-47). Chicago: RandMcNally.

Coleman, J. S., Menzel, H., \& Katz, E. (1957). Medical innovation: A diffusion study. Nova Iorque: Bobbs Merrill.

Flache, A., \& Macy, M. (1997). The weakness of strong ties: The collective action failure in a highly cohesive group. In P. Doreian \& F. Stockman (Eds.), Evolution of social networks (pp. 19-44). Amesterdão: Gordon and Breach.

Friedkin, N. E., \& Johnsen, E. C. (1990). Social influence and opinions. Journal of Mathematical Sociology, 15, 193-205.

Granovetter, M. (1973). The strength of weak ties. American Journal of Sociology, 78, 1360-1380.

Granovetter, M. (1982). The strength of weak ties: A network theory revisited. In P. V. Marsden \& N. Lin (Eds.), Social structure and network analysis (pp. 105-130). Londres: Sage.

Homans, G. (1951). The human group. New York: Harcourt Brace.

Maier, F. H. (1995). Innovation diffusion models for decision support in strategic management. In T. Shimada \& K. Saeed (Eds.), System dynamics (number 95, vol. 2). Tóquio: The System Dynamics Society.

Marsden. P. V., \& Friedkin, N. H. (1994). Network studies of social influence. In S. Wasserman \& J. Galaskiewicz (Eds.), Advances in social network analysis (pp. 3-25). Londres: Sage.

Nielsen, J. (1995). Bass curves for the diffusion of innovations. Nielsen-Norman Group, Alertbox, retirado da internet em www.useit.com.

Rogers, E. M., \& Kincaid, D. L. (1981). Communication networks: A new paradigm for research. Nova Iorque: Free Press.

Soczka, L., Pereira, E, Machado, P., \& Boavida, E. (1986). Children of Musgueira: A study on the social ecology of a shanty town. In D. Canter, J. Jesuino, L. Soczka, \& G. Stephenson (Eds.), Environmental social psychology (NATO ASI Series). Amesterdão: Kluwer Academic Press.

Soczka, L. (1989). Para uma perspectiva ecológica em psicologia. Lisboa: LNEC.

Soczka, L. (2001). Modelos de análise de redes sociais e limitações do modelo de equilíbrio estrutural de Heider. Psicologia, XV (1), 147-175.

Soczka, L. (2004). Contextos territoriais e perspectiva ecológica em psicologia social. In J. Vala \& M. B. Monteiro (Orgs.), Psicologia social. Lisboa: Fundação Calouste Gulbenkian.

Valente, T. (1995). Network models of the diffusion of innovations. Nova Jérsia: Hampton Press. 